\title{
MELIORATIONEN UND LANDSCHAFTSBILD IM KANTON ST. GALLEN ${ }^{1}$
}

\author{
VON HaNS BRASCHLER \\ Mit 13 Abbildungen und 1 Karte
}

Nach den großen Aufwendungen für Bodenverbesserungen während des Krieges 1939-1945 herrscht in unserem Lande vielfach die Auffassung, es sei nun alles durchmelioriert. Daß dem nicht so ist, soll im folgenden am Beispiel des Kantons St. Gallen gezeigt werden, wobei sich auch ergeben wird, wie stark die Kulturtechnik am Gestaltungswerk der Landschaft beteiligt ist.

In diesem vielgestaltigen, vom Mittelland über die Voralpen zu den Alpen reichenden Gebiet spielten Meliorationen von jeher eine wichtige Rolle. Von älteren Bemühungen abgesehen, wurden schon 1884 Güterzusammenlegungen im Anschluß an Bach-

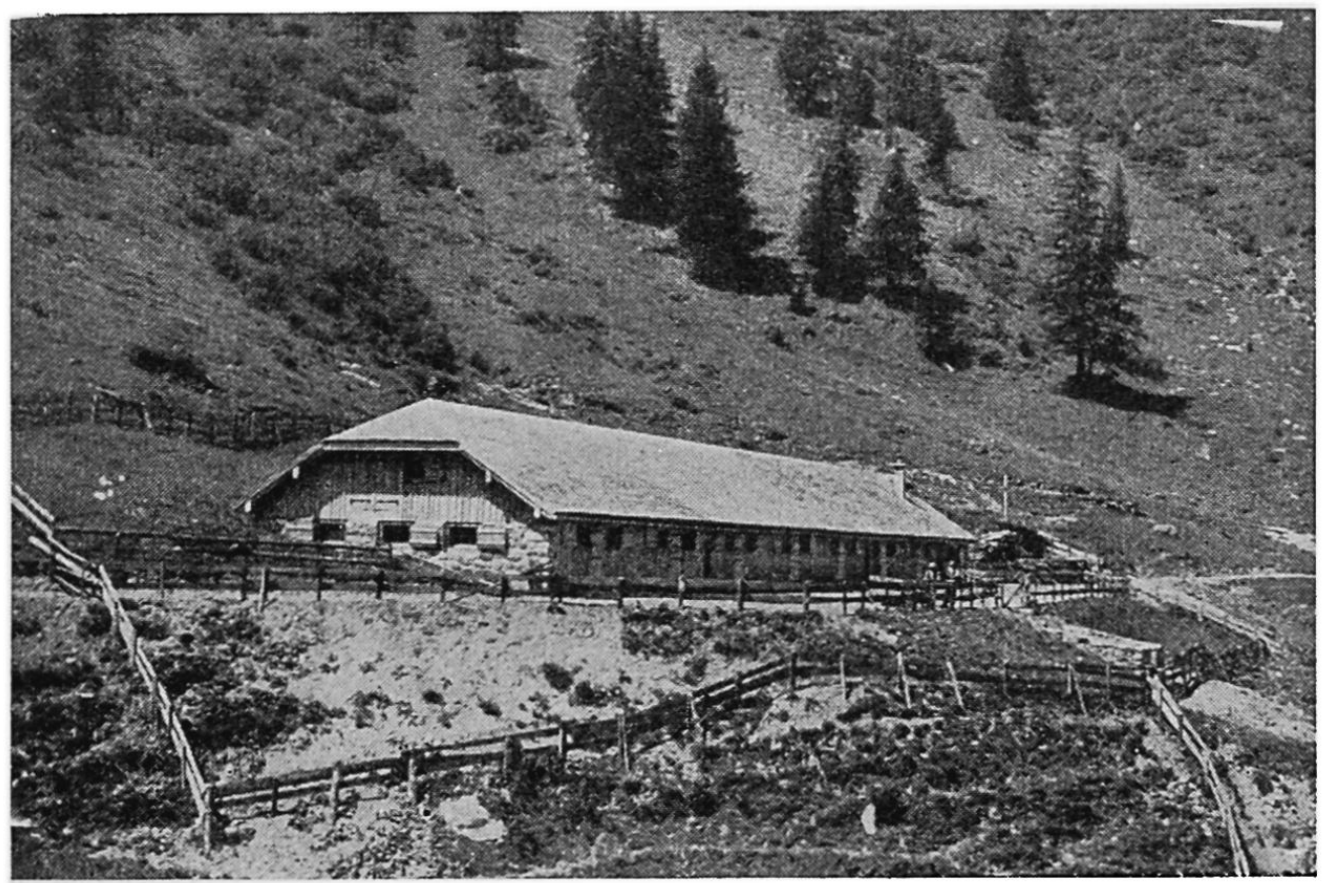

Abb. 1. Neuer Alpstall, Alp Kohlschlag, Mittelsäß, Gemeinde Mels. Querstall für 140 Stück Vieh. Erstellt 1947

korrektionen ausgeführt. In der folgenden Zeit traten sodann verschiedene weitere Formen von Meliorationen hinzu, die die Finanzen des Kantons - wie die von Bund, Gemeinden, Korporationen und Privaten - stark beanspruchten. Für Boden- und Alpverbesserungen allein wurden von 1884 bis 193925800000 Franken ausgegeben; während der letzten Kriegszeit kam eine Summe von 69996000 Franken hinzu, so daß gegenwärtig über 95 Millionen Franken in Meliorationen investiert sind.

Das Bodenverbesserungswesen wird seit 1898 vom kantonalen Kulturingenieur betreut. Bis 1944 war das kantonale Kulturingenieurbureau vom Vermessungsamt getrennt. Das erstere war dem Volkswirtschaftsdepartement, das letztere dem Baudepartement unterstellt. Um eine bessere Koordination beider Arbeiten und eine rationellere Personalverwendung zu erreichen, beschloß der Regierungsrat die Vereinigung dieser Dienststellen zu einem kantonalen Meliorations- und Vermessungsamt. Neben der Oberaufsicht über die in Durchführung begriffenen Meliorationen und die Begutachtung einlaufender Projekte übernimmt das Amt selber Projekte und bearbeitet hauptsächlich die generellen Projekte bei den Gesamtmeliorationen. Für die Projektierung und Bauleitung bestimmter Bodenverbesserungen werden verschiedene private Ingenieur- und Grundbuchgeometerbureaus beschäftigt.

1 Referat, gehalten an der Tagung der SNG St. Gallen 1948. Vgl. «Geogr. Helvetica» IV, 1948, S. 48. 


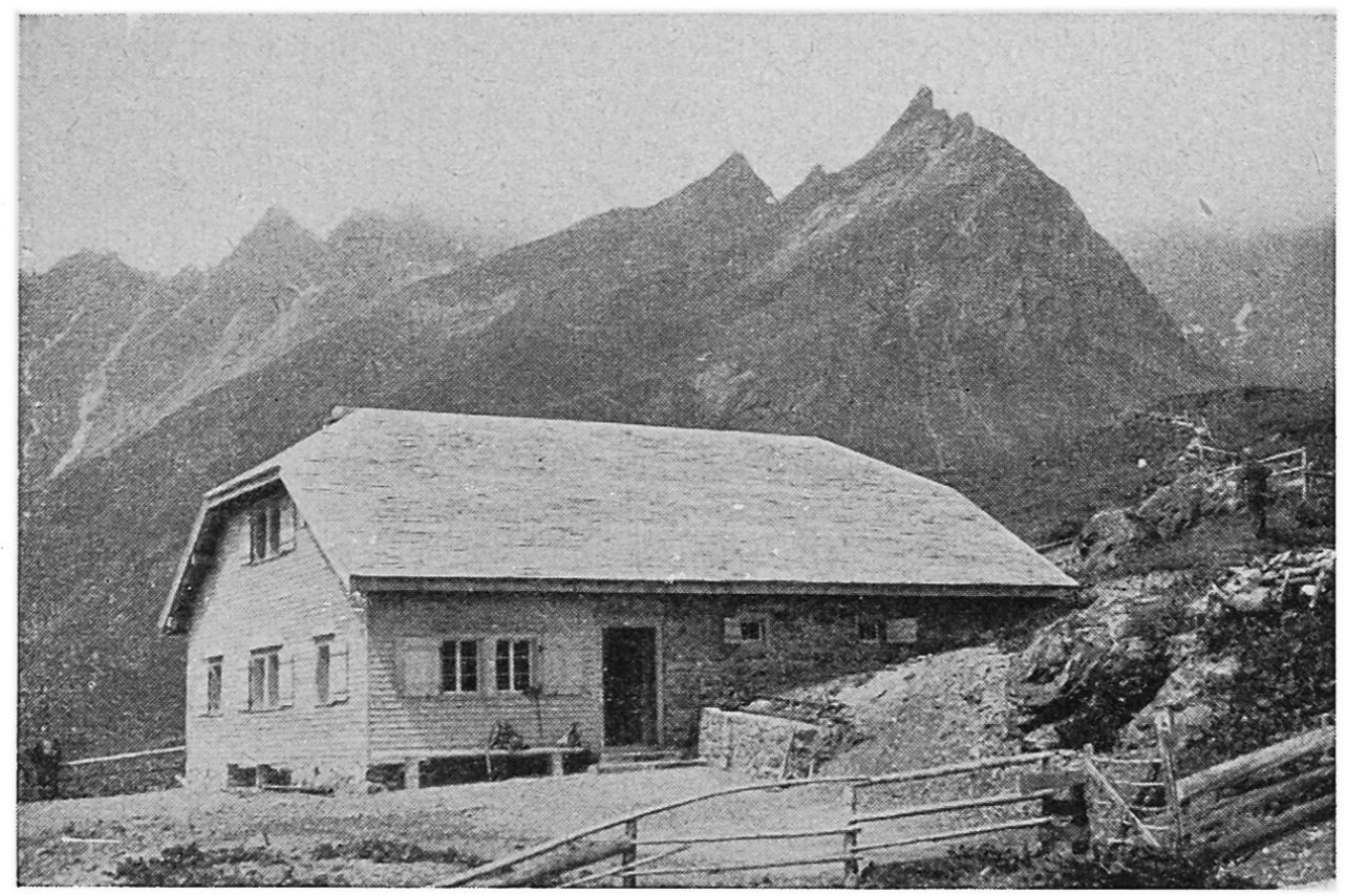

Abb. 2. Neue Sennhütte auf Alp Lasa 1947, Valens, erstellt 1947

Von den gegenwärtig in Ausführung begriffenen Werken sind vor allem die Melioration der Rheinebene mit einer Fläche von 6500 ha als größtes schweizerisches Gesamtmeliorationswerk und die der Linthebene, deren st.-gallischer Anteil 2928 ha um-

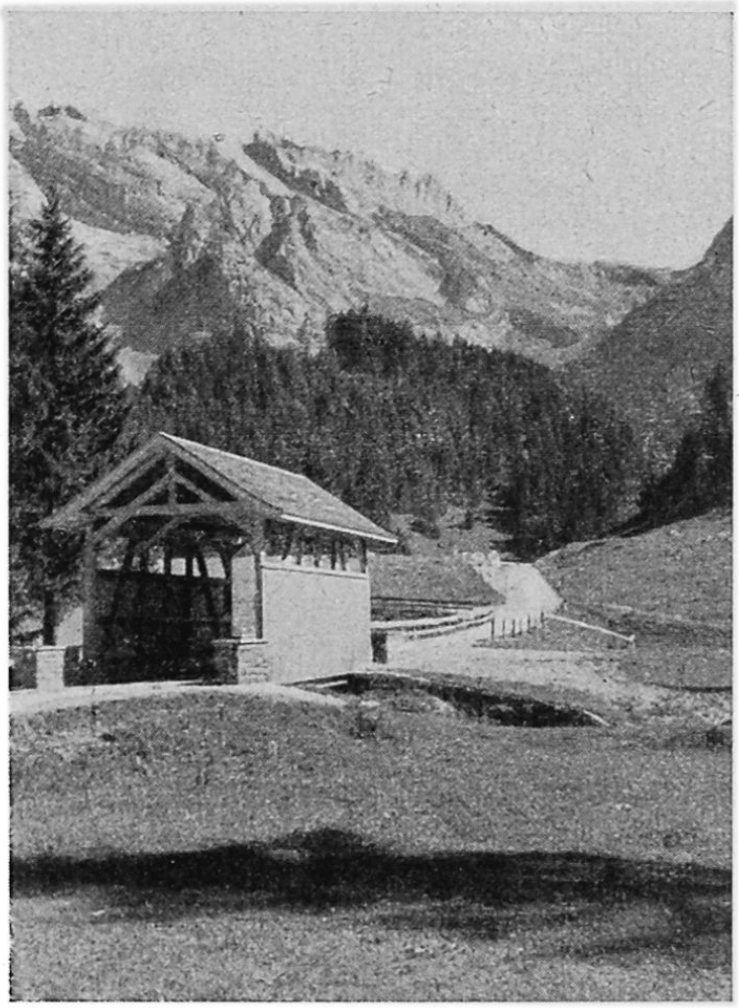

Abb. 3. Gedeckte Holzbrücke an der neuen Güterstraße Laui - Thurwies. Sie ist naturverbunden und fügt sich sehr gut in die prachtvolle Berglandschaft ein faßt, zu nennen. Neben diesen großen Werken sind die Alpverbesserungen von eminenter Bedeutung. Alpställe, Sennhütten, Düngerwege und Transportanlagen, ferner Wasserversorgungen, Räumungen usw. sind in den st.-gallischen Alpen neben den zahlreichen ausgeführten Objekten - an Alpställen und -hütten allein wurden 1884-1947 409 Projekte mit einem Kostenaufwand von 4965940 Franken realisiert — noch viele $z u$ erstellen (Abb. 1 und 2).

Auch Güterweganlagen sind schon viele gebaut worden: $1884-1947$ insgesamt 338 Objekte mit einem Kostenaufwand von 11547471 Franken (Abb. 3). Verschiedene ständig bewohnte Berggebiete entbehren aber noch immer derVerbindung mit dem Tal und müssen neu erschlossen werden. Hier liegen namentlich zahlreiche neue Projekte bereit, für den Fall einer drohenden Arbeitslosigkeit.

Nicht minder wichtig sind die Wasserversorgungen. Es ist klar, daß die Beschaffung von hygienisch einwandfreiem Trinkwasser in Alp- und ständig bewohnten Berggebieten zu einem der bedeutsamsten Probleme der Siedlungskorrekturen gehört und sie, da diese ebenfalls ein Bestandteil 


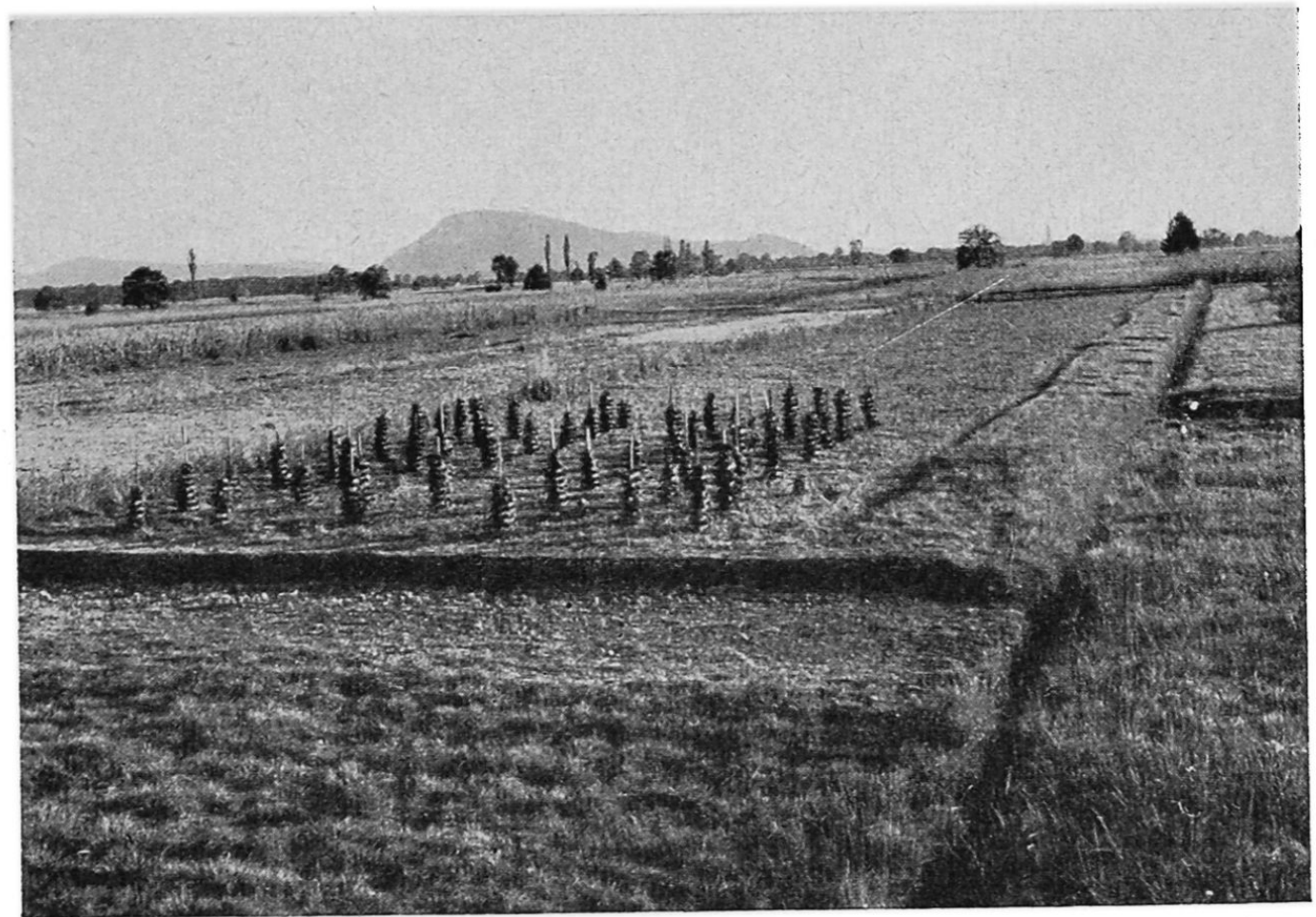

Abb. 4. Bernecker Ried vor der Melioration. Aufnahme vom 18. 9.1942. Wenig lohnende Torfausbeute und bescheidener Streuegewinn. Photo: ZumbüHL

des Aufgabenkreises des Kulturingenieurs darstellt, zweckmäßig ihm zugewiesen ist. Die Entwässerungen sind das, was man im Volk allgemein unter Melioration versteht. Meliorationen sind aber nicht nur Bodenverbesserungen im engern Sinne, sondern auch alle Maßnahmen, die zur Erleichterung der Bewirtschaftung dienen. Die 750 1884-1947 ausgeführten Drainagen erstrecken sich über eine Fläche von 5064 ha.

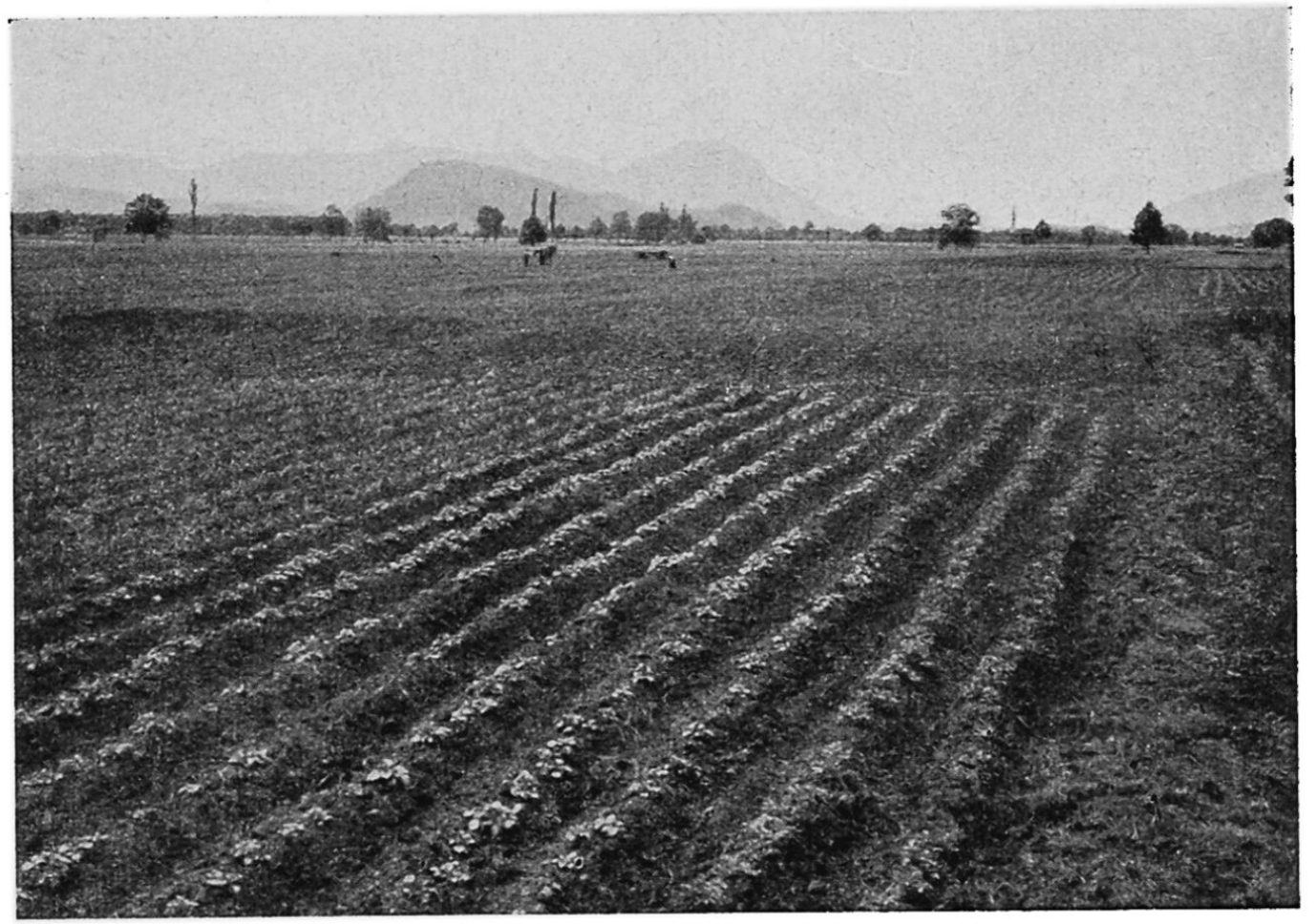

Abb. 5. Bernecker Ried nach der Melioration. Aufnahme vom 2.6.1943. Die ausgeführte Entwässerung ermöglichte lohnende Kartoffelkultur. Photo: ZuмвüHL 


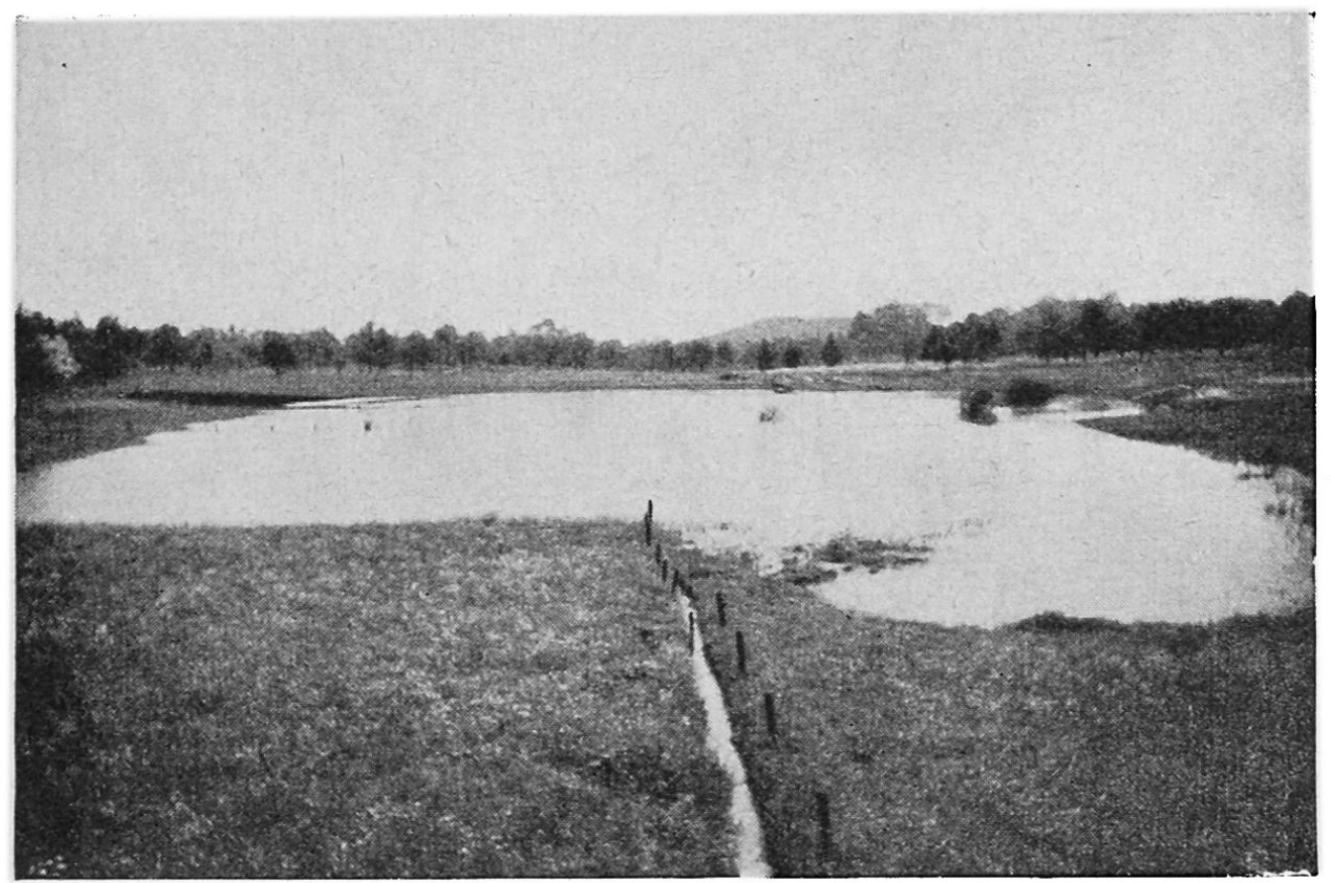

Abb. 6. Agener Moos vor der Entwässerung

Sie beanspruchten 10654209 Franken. In den verflossenen sieben Jahren wurde das $11 / 2$ fache dessen entwässert, was früher im Kanton St. Gallen während fünfzig Jahren ausgeführt wurde. Wenn der Hunger vor den Grenzen steht, dann sind die Meliorationen populär (Abb. 4-7).

Rodungen waren so gut wie ausschließlich Kriegsmaßnahme, und trotz vieler Kritik wurden und werden mittels ihrer respektable Mehrerträge erzielt. Irgendwo muß ja Realersatz geschaffen werden für das viele Land, das durch Bauten aller Art der landwirtschaftlichen Nutzung entzogen wird. In der Schweiz sind in den letzten 25 Jahren jedes Jahr 1000 Bauernbetriebe eingegangen. Im Kanton St. Gallen wurden während des letzten Krieges durch Festungs-Industrie- und Wohnbauten 433 ha beansprucht.

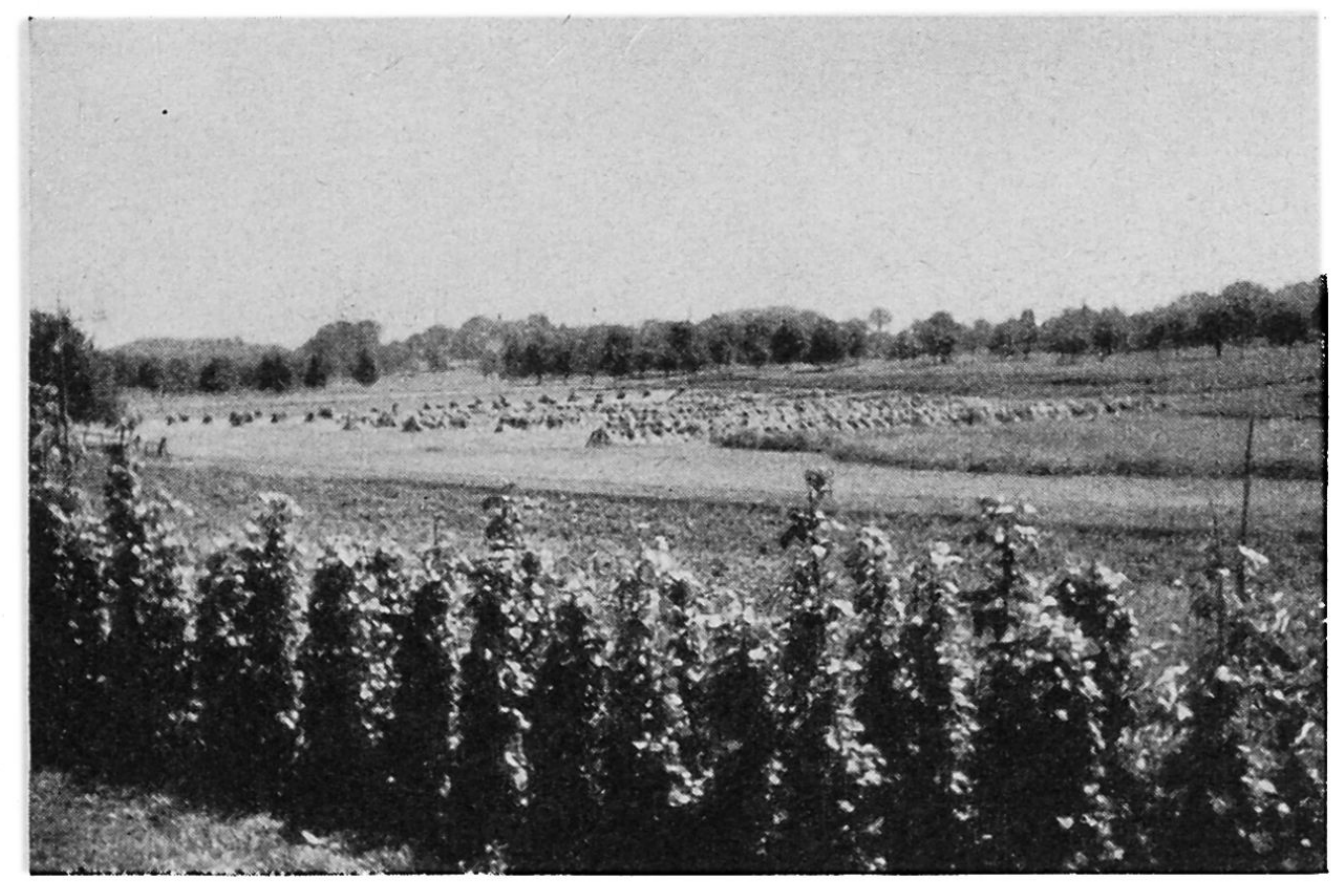

Abb. 7. Agener Moos, Kulturzustand im Sommer 1942 
Das sind 43 Familienbauernbetriebe. In diesem Zusammenhang sind auch die Rodungen $z u$ verstehen, die 1884-1947 im Kt. St. Gallen 116 Objekte auf einer Fläche von insgesamt 429 ha umfaßten (Abb. 8/9). Es ist jedoch klar, daß wir den Wald in Zukunft erhalten müssen, und deshalb werden Rodungen derzeit nicht mehr ausgeführt.Von großer Bedeutung sind ferner Rebbergverbesserungen. Insbesondere handelt es sich dabei um die Ausnützung guter Reb-

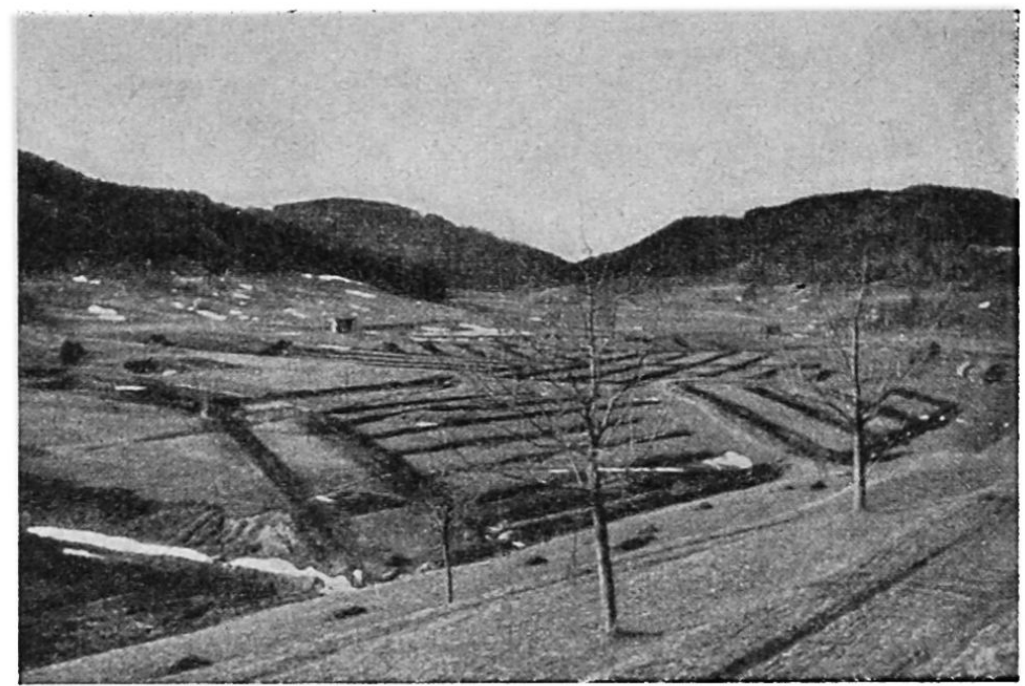

Abb. 8. Drainage im Bau. Botsberger Ried, Gemeinde Flawil lagen, in denen Weganlagen, Wasserversorgungen, Spritzanlagen und Rebbergzusammenlegungen zur Realisierung gelangten. Auch sie sind Aufgabe des Meliorationsdienstes. 1884-1947 wurden deshalb aus den Meliorationskrediten 268490 Fr. für Rebbergverbesserungen verwendet, wobei 19 Projekte zur Durchführung kamen (Abb. 10).

Luftseilbahnen sind überall dort zu erstellen, wo Straßenbauten zu teuer kommen und wo kein Zwischengelände erschlossen werden kann. Bisher (1884-1947) erfolgte die Realisierung von fünf Projekten im Baukostenbetrage von 114786 Franken.

Die Güterzusammenlegungen werden auch im Kanton St. Gallen inskünftig die bedeutungsvollste aller Meliorationen sein. 1884-1947 wurden hier 37 Projekte im Umfang von 4735 ha und im Kostenbetrage von 3818269 Franken ausgeführt, während noch rund 16800 ha Landes zusammenlegungsbedürftig blieben (Schweiz: rund 443000 ha). Die Schaffung weniger, aber großer und regelmäßiger Parzellen ist für unsere Landwirtschaft von größter Wichtigkeit. Die Rationalisierung der Betriebe ist eine Existenzfrage für unsern Bauernstand. Im Zusammenhang mit Güterzusammenlegungen müssen Weganlagen, Entwässerungen, Windschutzanlagen, Kanalisationen und Siedlungen projektiert werden. Auch die Fragen der Bebauung und die damit eng zusammenhängenden Aufgaben der Landesplanung sind zu lösen. Mit Preissteigerungen ist unserer Landwirtschaft nicht gedient. Wichtig ist, daß mit gleichem Aufwand mehr und besser produziert werden kann. Dies ist das wesentliche Ziel der Güterzusammenlegungen. Eine weitere bedeutsame, aber kostspielige Aufgabe ist die Besiedlung abgelegener Gebiete. Sie ist im gesamten Meliorationswerk von 1943-1949 mit 16 berufsbäuerlichen Siedelungen, 6 Trattställen

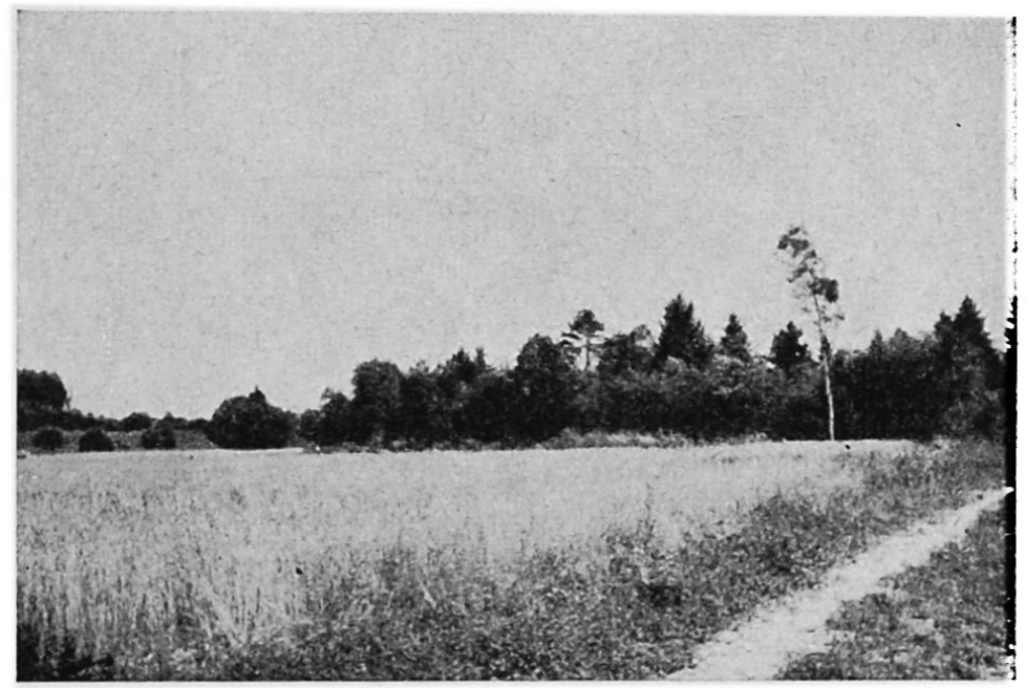

Abb. 9. Kornfeld auf Rodungsland an der Thur. Der notwendige Windschutz blieb bestehen 


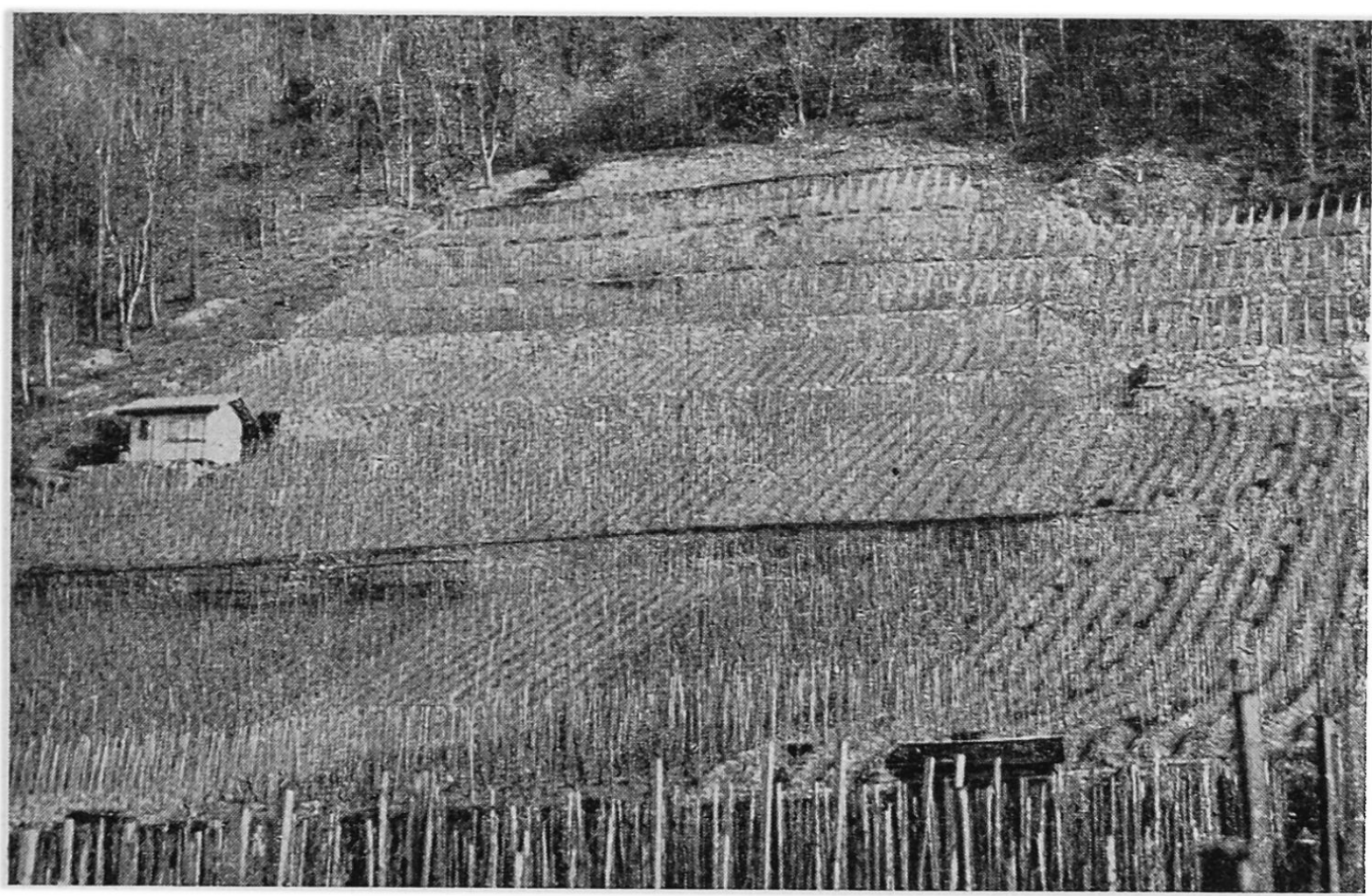

Abb. 10. Terrassenmauern in einem rekonstruierten Rebberg in Sargans

und einer Dienstbotenwohnung nebst Zuleitung von Wasser und Elektrizität mit einem Kostenbetrag von 2777030 Franken vertreten, woran der Kanton 672160 Franken an Beiträgen leistete. Alles Land, das noch zur Verfügung steht, muß intensiv bewirtschaftet werden. In dieser Beziehung stellen sich namentlich bei der Rhein- und Linthmelioration zahlreiche Probleme (Abb.11-13). - Ein Kuriosum ist die Hafen a nla ge

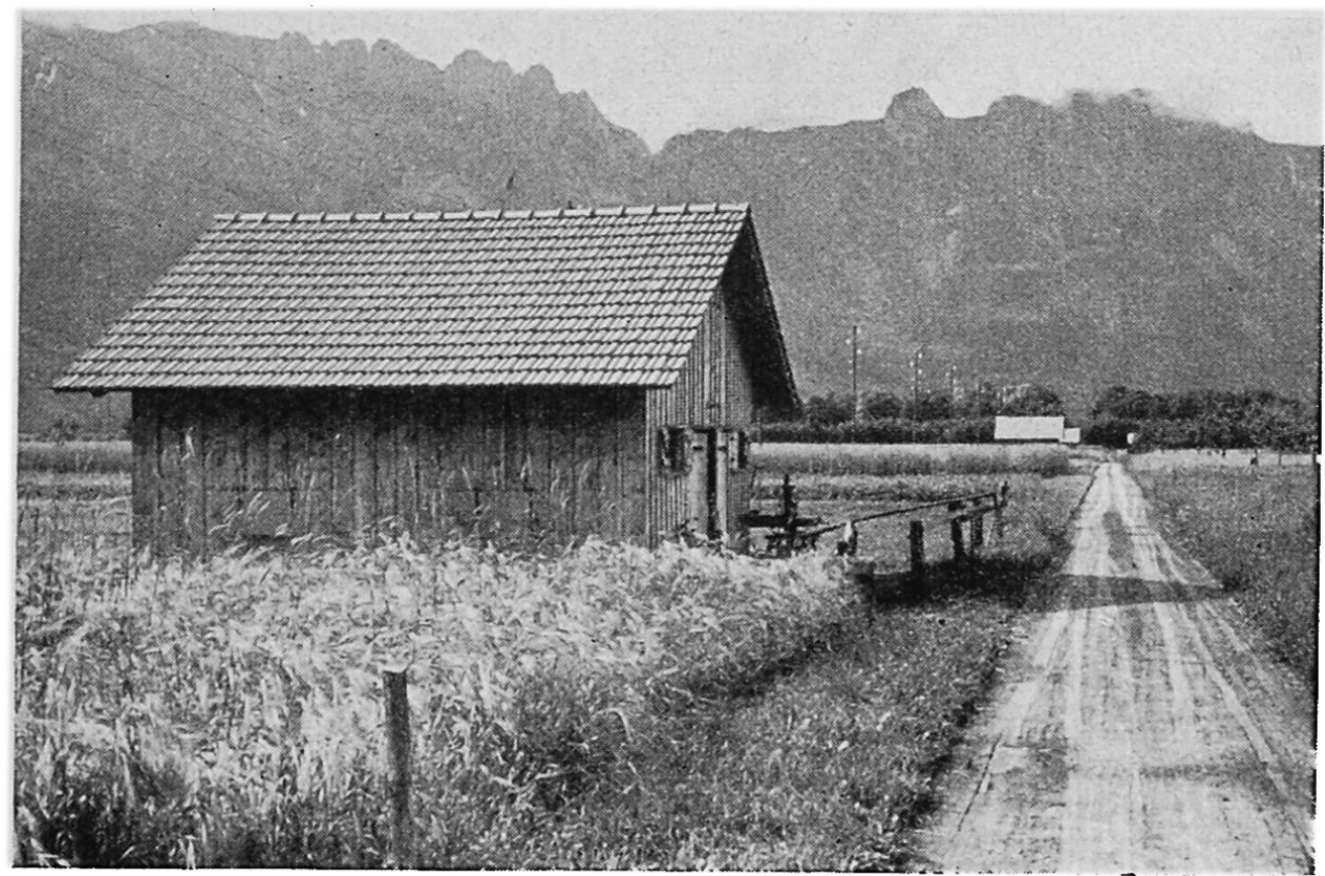

Abb. 11. Neu erstellte Feldscheunen und Weideställe im zusammengelegten Ortsgemeindeland Buchs (St.-Galler Rheintal) 


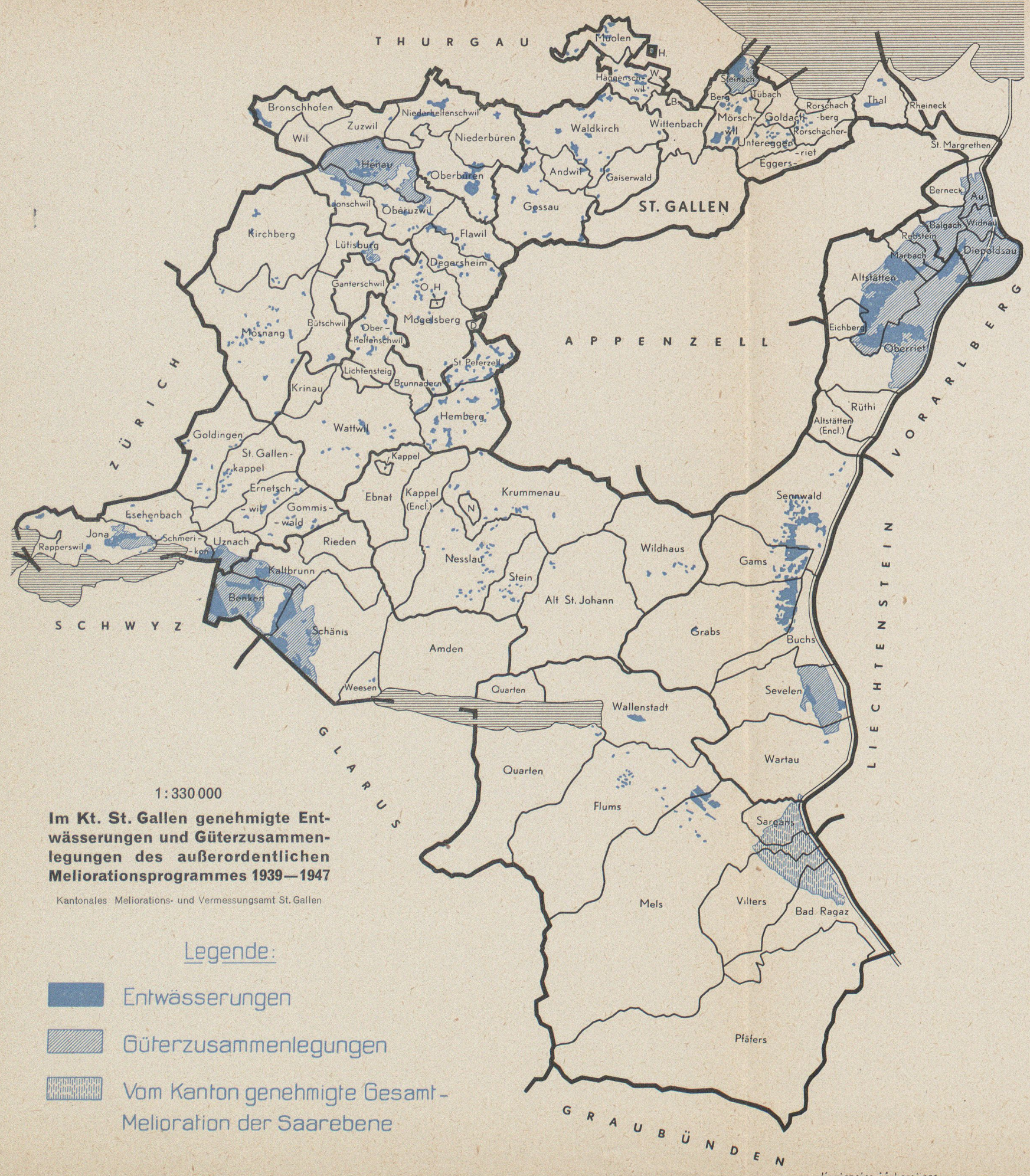






Abb. 12. Melioration der Rheinebene Altstätten. Alter Kesselbach als Windschutz und neuer Kesselbach mit Kiesfang und Parallelwegen. (Photo: Militärflugdienst Nr. 271/58) 


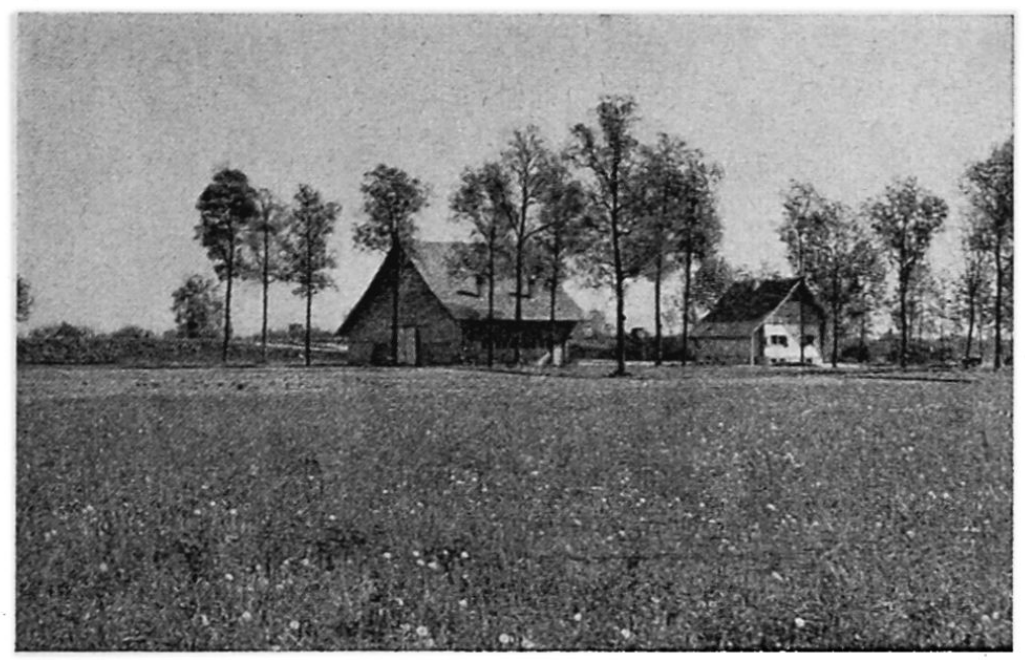

Abb. 13. Siedlung Fahrmad, Gemeinde Diepoldsau. Eingebettet in alte Bäume, steht dieser neue Hof auf Rodungsland an der Hohenemser Kurve in Quinten. Dieses Dörfchen ist auf den Seetransport angewiesen, was in der neuern Zeit die Verbesserung seines Hafens nötig machte. Die Erstellung und Unterstützung von Licht- und Kraftversorgungen in Berggebieten ist ebenfalls unsere Aufgabe. Zwischen 1884 und 1947 kamen im Kt. St. Gallen 100 Projekte mit $168329 \mathrm{~m}$ Freileitung im Kostenbetrag von 696564 Franken zur Ausführung. Ferner kommen Beregnungs- und $\mathrm{Be}$ wässerungsanlagen hinzu. Alle Werke müssen von

den betreffenden Melioranten gut und dauernd unterhalten werden. Oft werden aber gerade Alpgebäude und Straßen usw. durch höhere Gewalt, Lawinen, Stürme usw., zerstört und müssen von Zeit zu Zeit erneuert werden. Solche Rekonstruktionsarbeiten erfordern immer wieder eine Neuprojektierung, viel Zeit und Geld.

Aus dem Dargelegten, und insbesondere aus den Abbildungen, wird klar, welch große Wirkung Meliorationen auf die gesamte Landschaft, auf Natur- wie auf Kulturlandschaft, haben. Es wird dabei von den Behörden stets darauf geachtet, daß diese Wirkungen wenn immer möglich das Landschaftsbild nicht stören. Doch muß stets an alle Gutgesinnten unter den zuständigen Fachleuten appelliert werden, damit sie die breiteste Öfentlichkeit auch darüber aufklären, daß die Meliorationen nicht nur Vorkehren zum Durchhalten in Notzeiten bedeuten, sondern daß sie auch in Zukunft und für Friedenszeiten das bleiben werden, was sie immer waren: Maßnahmen zur Erleichterung der Bebauung des Bodens und zur Mehrung der so karg bemessenen Scholle unserer Heimat und damit Dienst an Land und Volk. Friedrich Der Grosse sagte: Wer bewirkt, daß dort, wo bisher ein Halm wuchs, deren zwei gedeihen, leistet mehr für sein Volk als ein Feldherr, der eine große Schlacht gewinnt.

Literatur: Das außerordentliche Meliorationsprogramm. Bericht über das Meliorationswesen der Schweiz 1940-1946. Bern 1947. - Das Meliorationswesen im Kanton St. Gallen während der Zeit des zweiten Weltkrieges 1939-1947. St. Gallen 1948. - H. Braschler: Zukunftsaufgaben für das Meliorationswesen im Kanton St. Gallen. S.-A. aus «St.-Galler-Bauer», Flawil 1948.

Die Klischees verdanken wir der Freundlichkeit des Chefs des Kantonalen Meliorations- und Vermessungsamtes St. Gallen, Dipl. Ing. H. Braschler.

\section{AMÉLIORATIONS FONCIERES ET PAYSAGE DANS LE CANTON DE SAINT-GALL}

La composition donne un aperçu sur les améliorations foncières exécutées dans le canton de SaintGall pendant les années 1884 à 1947. Elle traite surtout les formes principales suivantes: améliorations des alpages, constructions de routes et de chemins, adductions d'eau, assainissements, épierments, améliorations des régions viticoles, téléfériques, remaniements, colonisations rurales, conduites électriques et irrigations.

\section{MELIORAZIONI FONDIARIE E PAESAGGIO NEL CANTONE DI SAN GALLO}

La composizione dà una vista d'occhio sulle meliorazioni eseguite nel periodo. 1884 a 1947 nel cantone di San Gallo. Sono trattate anzitutto le forme principali, cioè: bonifica dei alpi, costruzione di strade e sentieri, acquedotti, prosciugamenti, spietramenti, bonivica delle vigne, teleferiche, raggruppamento terreni, colonizzazioni rurali, condotte elettriche, irrigazioni. 